\title{
Seismic noise study for a new seismic station at King Fahd University of Petroleum and Minerals in Saudi Arabia
}

\author{
S. I. Kaka \\ Earth Sciences Department, KFUPM, Dhahran 31261, Saudi Arabia \\ Correspondence to: S. I. Kaka (skaka@kfupm.edu.sa)
}

Received: 28 October 2012 - Revised: N.A. - Accepted: 4 December 2012 - Published: 30 April 2013

\begin{abstract}
In this paper, I describe the work undertaken at King Fahd University of Petroleum and Minerals (KFUPM) in Saudi Arabia to select a suitable site for a new broad band seismic station. The new station will be equipped with a 3-component $120 \mathrm{~s}$ to $50 \mathrm{~Hz}$ Trillium 120 broad band seismometer, Taurus 24-bit data acquisition system along with a large LCD to display the waveform data in real-time. The KFUPM community will have an opportunity to observe daily seismic activity in real-time and to monitor/record both regional and teleseismic events. Moreover, students will gain the opportunity to identify P, S, Love, and Rayleigh waves and learn how to locate an earthquake. The station will also play an important role in providing a source of information about seismic activity for the general public. The station is expected to be operational in a few weeks time.
\end{abstract}

\section{Introduction}

It is well known that setting up a seismic station, specifically the location of the seismic station, depends on the signal and noise characteristics. Hence, selecting a quiet site with low seismic noise and with good signal-to-noise ratio is necessary to ensure that the station is capable of detecting earthquakes while recording representative waveforms. If the station is located at a noisy site, we need to configure higher trigger thresholds and it will likely result in poor detestability. In fact, understanding the noise level is essential for choosing a suitable location for a seismic station (UCB SL, 1997). Consequently, I have carried out a seismic noise study in order to understand the noise level at various selected locations on the KFUPM campus, Dhahran, Saudi Arabia. The main purpose is to select a suitable site with low seismic noise and good signal-to-noise ratio for a new broadband seismic station.

\section{Seismic noise monitoring system}

The seismic noise monitoring system used in this study was manufactured by OYO Geospace (http://www.geospacetech. $\mathrm{com} /$ ). The system was recently acquired through a project funded by the National Science, Technology and Innovation Plan of the King Abdulaziz City for Science and Technology (KACST) program and includes a seismic recorder and a sensor (geophone) with a GPS device. After calibrating and testing the system, it was deployed to measure and monitor the noise at various locations of interest. The system continuously measures and monitors seismic noise for twenty eight days (based on the memory available with the system). This continuous monitoring was done as part of a larger study where passive seismic recordings were made to better understand and characterize the origin of various nearsurface noises over a non-producing reservoir in Dhahran, Saudi Arabia (Papoola and Kaka, 2011; Kaka, 2012). A total of fifteen $10 \mathrm{~Hz} 3$-Component geophones with $4 \mathrm{~ms}$ sampling rate corresponding to sampling frequency of $250 \mathrm{~Hz}$ were used in this study.

\section{Study area and site selection}

\subsection{Study area}

The study area (i.e. noise monitoring stations) is located on the KFUPM campus in Dhahran, a city located in Saudi Arabia's eastern province. The study area was chosen due to its accessibility and proximity to the department of Earth Sciences (ESD) at KFUPM. The campus is situated at an elevation of $150 \mathrm{~m}$ on top of a hill known as Jebel Umm Er Rus. The Jebel Umm Er Rus is part of an extremely gentle sloping Dammam Dome which represents a hydrocarbon structural trap. The exposed rocks at our monitoring area consist 


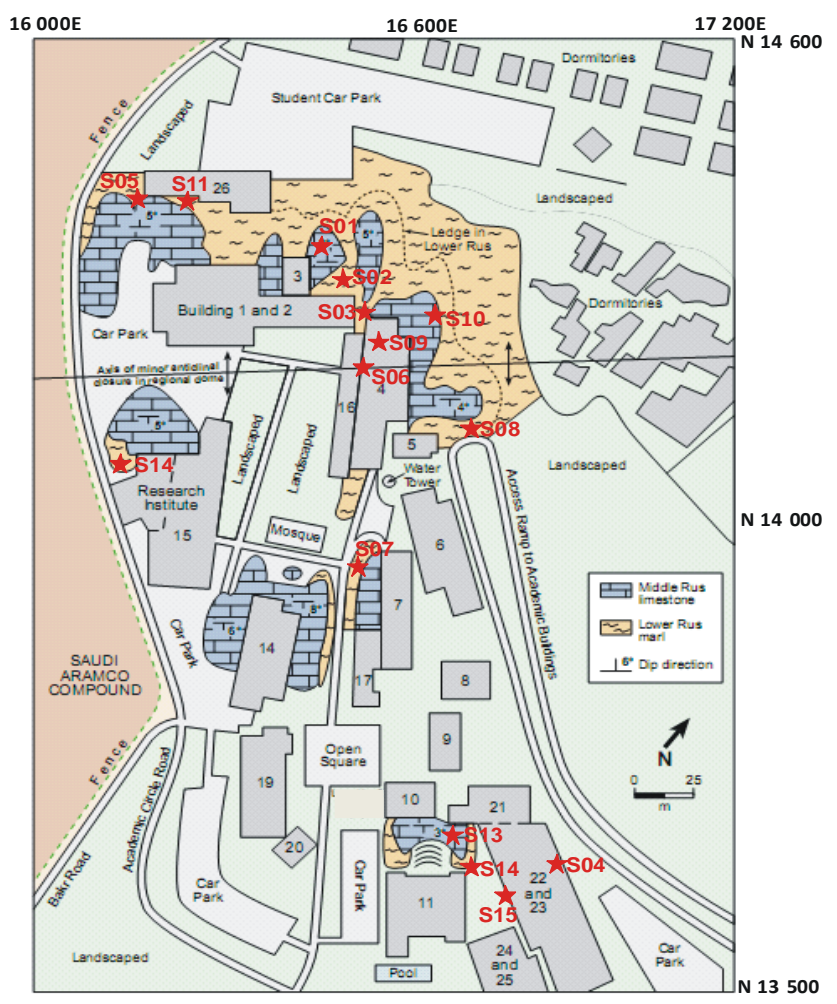

$\star$ Recording Stations

Fig. 1. Map showing KFUPM campus and the location of geophones (modified after Weijermars, 1999).

of three different units of the Rus Formation (i.e. lower Rus, middle Rus and upper Rus). The monitoring stations were distributed over all three Rus Formation units. The lower Rus, a $21 \mathrm{~m}$ thick unit, is composed of grey to buff, partly dolomitized limestone while the middle Rus, $31.8 \mathrm{~m}$ thick, is composed of alternating light-colored marl and limestone beds, and the upper Rus, consists of $3.6 \mathrm{~m}$ thick white, soft chalky, porous limestone (for further details on surface geology and lithostratigraphy, see Weijermars, 1999). This study is intended to be the first of many at KFUPM, and we will attempt to investigate the characteristics of passive signals from all three Rus units during the second phase of this study.

\subsection{Site selection}

There are several factors involved in the selection of a site location for a new station. Most importantly, a balance needs be be struck between a logistically convenient site versus a technically suitable site. As a starting point, fifteen potential sites were selected across KFUPM campus. Figure 1 shows the KFUPM campus and the locations of monitoring sites. Sites that are relatively close to possible low-frequency noise sources were then eleminated. Many possible noise sources were considered, including: vehicle traffic/heavy machinery,direct path of air flowing from air conditioning vent, tall

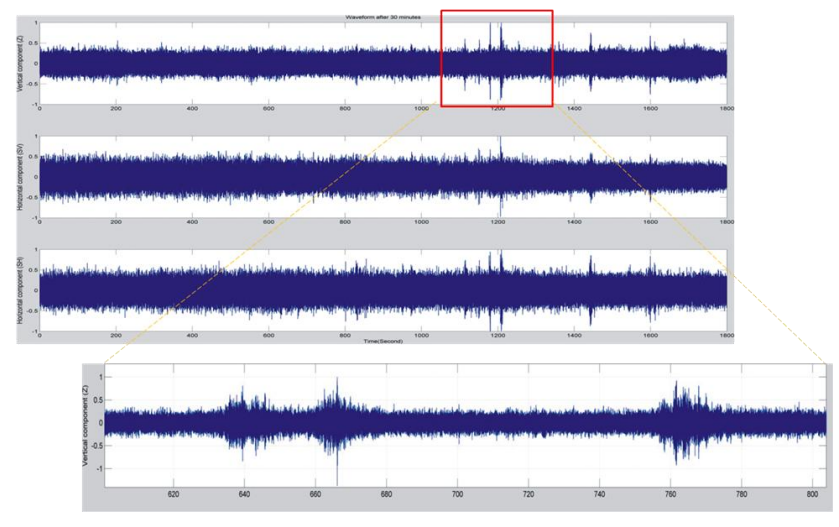

Fig. 2. Thirty minutes of the recorded time series data. The zoomedin part of the vertical component data shows larger amplitude as well as discontinuities.
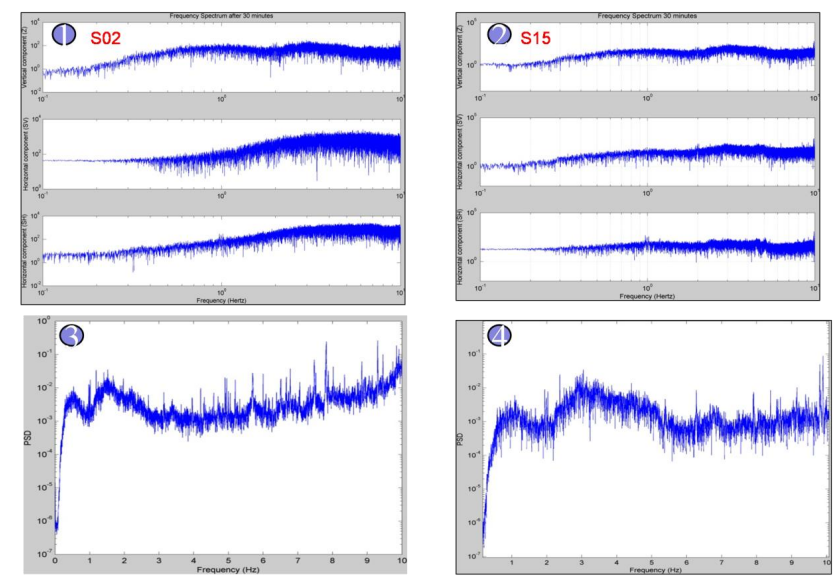

Fig. 3. Frequency spectrum of stations S02 and S15 (1 and 2) with corresponding PSD (3 and 4).

trees/power poles and metal doorways. One more site was eliminated because it was located in the open area where it experiences maximum wind speed that is considered a major source of noise. Finally, data from five potential sites ( $\mathrm{S} 02$, S03, S04, S09 and S15) distributed at various location on the KFUPM campus were analyzed. S02 is located in the upper Rus formation, S03 and S09 are in the middle Rus formation and S04 is also located within the middle Rus formation but in a location less affected by the effects of human activities and strong wind. While S15 is located on a concrete foundation structure which used to house the seismic station that is no longer functional (Fig. 1). The study area is not known for any major faults and considered to have very low seismicity and hence the identification of seismoteconic features is not required. 


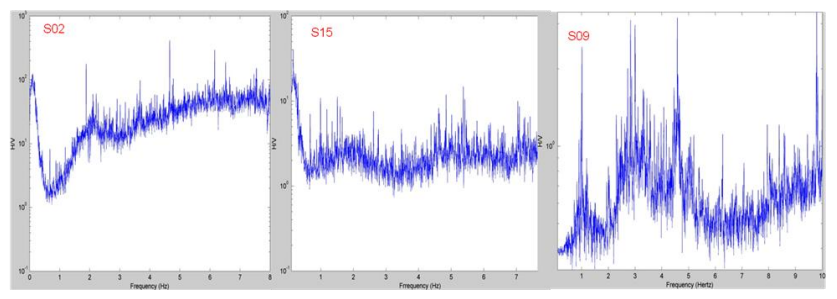

Fig. 4. The H/V spectral ratio at stations S02, S09 and S15.

\section{Data processing, analysis, results and discussions}

\subsection{Processing and analysis}

A continuous time-series data recorded by all five stations was visually inspected and it was found that the data was noisy during the day mainly due to vehicle traffic and heavy construction machineries that are constantly operating at nearby locations. Thus, I decided to process data recorded at midnight when all the above mentioned noise sources is minimal and only noise from the air conditioning system is expected to contribute to the noise, and hence can be easily identified. Figure 2 shows the typical example of the recorded data displayed as a time series. Both larger amplitude and discontinuities of the data could be observed in the vertical component. A similarity observed between the $\mathrm{SH}$ and SV waveforms thus indicate a fair homogeneity in the near surface. Over the years, many researchers used various spectra analysis, including Nakamura's method (Lermo and Chavez-Garcia, 1994). However, I found that the spectra peaks are not sharp over a certain frequency and often cover a considerably wide range of frequencies. I therefore employed the power spectra density (PSD) as an additional tool in our analysis to further enhance the peaks of the dominant frequency in the data. This will enable us to identify the peaks and remove ambiguities associated with the spectra peaks. Consequently, time series data were processed to generate the standard spectral, power spectral density (PSD) and H/V plots (Lermo and Chavez-Garcia, 1994; Saenger et al., 2009).

\subsection{Results and discussions}

The frequency spectratrum of stations S2 and S15 are shown in Fig. 3.1 and 3.2 and the corresponding PSD is plotted in Fig. 3.3 and 3.4. The spectral characteristics are clearer on the PSD plot and it is assumed that there is no fundamental difference between the Fourier transformed spectral and PSD. Spectral peaks are observed in the frequency range of $2-3 \mathrm{~Hz}$ and sometimes up to around $6 \mathrm{~Hz}$ (Fig. 4). These peaks are likely caused by near-surface site dependent effects. A recent study of borehole passive seismic data in a nearby area attributed the particle motion of the event to a preferred direction (Vesnaver et al., 2011) which is believed to have been caused by surface waves. Since all our record- ing stations are on soft rocks with respect to the subsurface rock density, it is likely that the observed spectral peaks are due to near surface site response. The characteristics of the noise using Fourier amplitude spectra and their corresponding spectrogram show that at frequencies greater than $1 \mathrm{~Hz}$, the noise sources seem to have cultural origin. We also observed variations of noise levels at one particular station. I belive that it is due to the proximity of the pedestrian crossing between two academic buildings. The results also show that at frequencies 0.05 to $1 \mathrm{~Hz}$, the level of noise is more uniform among three stations though the station close to the pedestrian crossing show a slightly higher level. As expected, horizontal components have a higher noise level than that of vertical components. Figure 4 shows the H/V spectral ratio at stations S02, S09 and S15. Both S02 and S15 are found to be at a slightly lower noise level then S09. Moreover, these sites receive minimal exposure to direct sunlight and thus thermal stability is provided. Consequently, it is recommended that the future station be installed at either S02 or S15 depending on a logistically convenient site.

\section{Conclusions}

It is well known that fairly good contact between seismometer and bedrock is one of the requirements for installing a seismic station. Interaction of local soil can significantly modify seismic signals and their spectral properties. Thus, it is recommended to install the station on S15 as it is located on a concrete foundation structure that goes all the way to the bedrock. The new seismic station will be a primary station in the eastern city of Saudi Arabia and will provide information on local earthquakes. More importantly, the station will play an essential role in providing an opportunity to educate the students and general public about earthquakes and ground motions.

Acknowledgements. The author would like to acknowledge the continuing support provided by King Fahd University of Petroleum and Minerals. The author also thanks Abdulaziz Al-Shaibani and Abdulatif Al-Shuhail for their encouragement and Qazi Sohail and Afeez Papoola for their help during data acquisition and processing. The author also acknowledge the National Science Technology and Innovation Plan for funding this work through the project \# 08OIL81-4.

\section{References}

Kaka, S. I.: Passive seismic experiment at King Fahd University of Petroleum and Minerals in Saudi Arabia, Seismol. Res. Lett., 83, 680-685, 2012.

Lermo, J. and Chavez-Garcia, F. J.: Are Microseismic Useful in Site Response Evaluation?, Bulletin of Seismological Society of America, 84-85, 1350-1352, 1994.

Papoola, A. and Kaka, S. I.: Microtremor Measurements Over a Non-Producing Reservoir in Dhahran, Saudi Arabia, the AGU 
Fall Meeting, 5-9 December 2011, at the Moscone Convention Center, San Francisco, California, Abstract control \#1186024, 2011.

Saenger, E. H., Stefan, M. S., Lambert, M., Nguyen, T. T., Torres, A., Metzger, S., Habiger, R. M., Muller, T., Rentsch, S., and Mendez-Hernandez, E.: A Passive seismic survey over a gas field: Analysis of low-frequency anomalies, Geophysics, 74, O29-40, 2009.

UCB SL: Guidelines for installing broadband seismic instrumentation, available at: ftp://quake.geo.berkeley.edu/outgoing/ installation/bi_guide.ps.gz (last access: August 2008), 1997.
Vesnaver, A., Menanno, G., Kaka, S. I., and Jervis, M.: 3D polarization analysis of surface and borehole microseismic data, Expanded Abstracts, SEG International Exposition and EightyFirst Annual Meeting, (San Antonio, Texas), Reference No. 475, 2011.

Weijermars, R.: Surface geology, lithostratigraphy, and Tertiary growth of the Dammam Dome, Saudi Arabia. A new guide, GeoArabia, 4-2, 199-266, 1999. 